\title{
负偏压下 $(\mathrm{Ni}-\mathrm{Mo}) / \mathrm{TiO}_{2}$ 膜电极光电催化降解罗丹明 $\mathrm{B}$ 的性能和机理
}

\author{
李爱昌*, 李健飞 ${ }^{b}$ 刘亚录 ${ }^{a} \quad$ 张建平 $^{a}$ 赵丽平 ${ }^{a}$ 卢艳红 $^{a}$ \\ ( ${ }^{a}$ 廊坊师范学院化学与材料科学学院 河北廊坊 065000) \\ ( ${ }^{b}$ 石家庄学院 石家庄 050035)
}

\begin{abstract}
摘要 用复合电沉积方法制备了 $(\mathrm{Ni}-\mathrm{Mo}) / \mathrm{TiO}_{2}$ 薄膜电极, 以扫描电子显微镜 $(\mathrm{SEM}) 、 \mathrm{X}$ 射线衍射 $(\mathrm{XRD})$ 、拉曼光谱 (Raman Spectra)和紫外-可见漫反射光谱(DRS)对薄膜的表面形貌、晶相结构和光谱特性进行了表征，在负偏压和可见 光作用下, 以罗丹明 $\mathrm{B}$ 为模拟污染物研究了薄膜的光电催化性能. 采用电化学技术和向溶液中加入活性物种捕获剂的 方法对薄膜光电催化降解机理进行了探索. 结果表明: $(\mathrm{Ni}-\mathrm{Mo}) / \mathrm{TiO}_{2}$ 薄膜是由粒径为 $50 \sim 100 \mathrm{~nm}$ 的 $\mathrm{TiO}_{2}$ 纳米粒子相和 纳米晶 $\mathrm{Ni}-\mathrm{Mo}$ 固溶体相构成的复合薄膜. 薄膜具有较高的光电催化活性, 在 $-0.4 \mathrm{~V}$ 偏压和可见光照射下反应 $60 \mathrm{~min}$, 复合薄膜光电催化罗丹明 $\mathrm{B}(c=5 \mathrm{mg} / \mathrm{L})$ 的降解率是多孔 $\mathrm{TiO}_{2}(\mathrm{P} 25) / \mathrm{TTO}$ 纳米薄膜的 1.56 倍. 复合薄膜电极中 $\mathrm{Ni}-\mathrm{Mo}$ 纳 米晶合金对溶解氧和激发电子还原反应的催化作用是光电催化降解活性提高的重要原因. 通过调节外加偏压, 可以控 制电极溶液界面间染料与活性氧化物种的存在形式及其相互作用, 是研究可见光催化降解反应历程的有效方法. 在负 偏压和可见光作用下, 羟基自由基和染料正离子自由基对染料的光电催化降解有决定性作用.
\end{abstract}

关键词 $(\mathrm{Ni}-\mathrm{Mo}) / \mathrm{TiO}_{2}$ 薄膜; 光电催化; 负偏压; 罗丹明 $\mathrm{B}$; 机理

\section{Photoelectrocatalytic Properties and Reaction Mechanism of $(\mathrm{Ni}-\mathrm{Mo}) / \mathrm{TiO}_{2}$ Film Electrode for Degradation of Rhodamine B at Negative Bias

\author{
Li, Aichang*,a Li, Jianfei ${ }^{b} \quad$ Liu, Yalu $^{a} \quad$ Zhang, Jianping ${ }^{a}$ \\ Zhao, Liping $^{a} \quad$ Lu, Yanhong ${ }^{a}$ \\ ( ${ }^{a}$ Faculty of Chemistry and Material Science, Langfang Teachers College, Langfang 065000, China) \\ $\left({ }^{b}\right.$ Shijiazhuang University, Shijiazhuang 050035, China)
}

\begin{abstract}
In the purpose of preparing highly efficient film photocatalyst and exploring rule of semiconductor photocatalytic and photoelectrocatalytic degradation of organic pollutants, $(\mathrm{Ni}-\mathrm{Mo}) / \mathrm{TiO}_{2}$ composite thin films were prepared by composite electroplating at a constant current. The depositing conditions of $(\mathrm{Ni}-\mathrm{Mo}) / \mathrm{TiO}_{2}$ film were as follows: suspension quantity of $\mathrm{TiO}_{2}$ in solution was $6.0 \mathrm{~g} / \mathrm{L}$; $\mathrm{pH}$ value in solution was 10.5 ; electrodepositing time and current density were 15 min and 95 $\mathrm{mA} / \mathrm{cm}^{2}$, respectively. The surface morphology, phase structure, and spectral characteristics of the thin film were analyzed by scanning electron microscopy (SEM), X-ray diffraction (XRD), Raman spectrum and ultraviolet-visible diffuse reflectance spectroscopy (DRS), respectively. Its photoelectrocatalytic properties were evaluated with Rhodamine B as a model compound under the conditions of negative bias and visible light. Using electrochemical technique and adding active species scavenger to the solution, the mechanism of photoelectrocatalytic degradation for the film were explored. The results indicate that the $(\mathrm{Ni}-\mathrm{Mo}) / \mathrm{TiO}_{2}$ film consists of crystalline grains of $\mathrm{TiO}_{2}$ in the size range of $50 \sim 100 \mathrm{~nm}$ and the nano-crystalline of $\mathrm{Ni}-\mathrm{Mo}$ in solid solution. The (Ni-Mo)/ $/ \mathrm{TiO}_{2}$ film is photoelectrocatalytically more active than $\mathrm{TiO}_{2} / \mathrm{ITO}$ (indium tin oxide) film. Reacting $60 \mathrm{~min}$ under the negative bias of $-0.4 \mathrm{~V}$ and visible light irradiation, the photoelectrocatalytic degradation rate of $(\mathrm{Ni}-\mathrm{Mo}) / \mathrm{TiO}_{2}$ film is 1.56 times as much as that of porous $\mathrm{TiO}_{2}($ Degussa $\mathrm{P} 25) / \mathrm{ITO}$ film. The improvement in photoelectrocatalytic degradation activity for the composite film could be mainly attributed to the catalysis of Ni-Mo in the composite film for the reaction of excited electrons with oxygen. By adjusting the applied bias, the existing forms of the dye and active oxygen species at the electrode/electrolyte surface and their interactions can be controlled. It is the efficient method to study the visible light catalytic degradation reaction mechanism. Both the hydroxyl radical and the dye cationic radical have a decisive role on the photoelectrocatalytic degradation of dyes under the conditions of negative bias and visible light.

Keywords (Ni-Mo)- $\mathrm{TiO}_{2}$ film; photoelectrocatalysis; negative bias; Rhodamine B; mechanism
\end{abstract}

* E-mail: aichangli@hotmail.com; Tel.: 0316-2188370

Received March 4, 2013; published April 10, 2013.

Project supported by the Science and Technology Project of Hebei Province (No. 11276732) and the Scientific Research Program of Langfang Teachers College (No. LSZZ201202).

项目受河北省科技支撑计划项目(No. 11276732)和廊坊师范学院科学研究项目(No. LSZZ201202)资助. 


\section{1 引言}

$\mathrm{TiO}_{2}$ 因其光稳定性、高效性、无毒和廉价等优点被 认为是具有良好应用前景的光催化剂 ${ }^{[1]}$, 被广泛用于有 机物废水的研究中 ${ }^{[2,3]}$. 为了使光催化处理后 $\mathrm{TiO}_{2}$ 与水 分离和回收再利用, 当前人们多采用负载型催化剂, 其 中主要是薄膜(层)负载催化剂来解决 ${ }^{[4]}$. 为了提高催化 效率, 许多研究者对纯 $\mathrm{TiO}_{2}$ 纳米薄膜表面进行改性修 饰, 特别是金属表面修饰, 并且由此出发逐步转向复合 薄膜的制备, 从而使对 $\mathrm{TiO}_{2}$ 薄膜的修饰由表面发展到 膜内. Hosseini 等 ${ }^{[}$把银纤维固定在不锈钢基片上, 用电 泳法制备了 $\mathrm{TiO}_{2}$ 质量分数为 $11 \%$ 的 $\mathrm{Ag} / \mathrm{TiO}_{2}$ 复合薄膜, 并研究了光催化降解亚甲基蓝的性能. 更多的文献以非 金属碳(活性炭和碳纳米管)和金属镍作为复合物制备 $\mathrm{TiO}_{2}$ 复合薄膜 ${ }^{[6,7]}$.

为了进一步提高光量子产率, 近年来研究者们提出 了一种将外加电场引入光催化反应体系的光电催化技 术. 在外加电场的驱动下, 光照 $\mathrm{TiO}_{2}$ 所产生的光生空穴 和电子向相反方向运动, 从而抑制光生电荷的复合, 提 高光催化量子产率和对污染物的降解效率. 大多数研究 者关注紫外光照射下外加正偏压对有机污染物的降 解 ${ }^{[8,9]}$. 事实上, 对有机染料的可见光催化降解, 可以在 一定负偏压下快速进行. 不仅如此, 在光催化体系中, 更重要的是可以通过外加偏压控制电极表面自由基的 形成 ${ }^{[10,11]}$, 从而研究有机污染物在不同偏压下的催化降 解途径, 以提高降解效率. 例如在一定负偏压作用下, 可以确保只有超氧自由基(通过还原氧气)在 $\mathrm{TiO}_{2}$ 电极表 面形成 ${ }^{[12]}$. 最近, 赵进才等 ${ }^{[12 \sim 15]}$ 开展了在负偏压和可 见光作用下, 用 $\mathrm{TiO}_{2}$ 膜电极光电催化降解有机染料的 研究工作. 研究结果表明, 可见光光电催化反应存在一 个最佳负偏压, 通过对此偏压下实验结果的分析, 可以 揭示光催化反应机理.

为了制备高效薄膜光催化剂, 本研究组把 $\mathrm{TiO}_{2}$ 纳 米微粒的金属表面修饰和固定化集于一身, 用电沉积方 法在紫铜片上制备了 $(\mathrm{Ni}-\mathrm{Mo}) / \mathrm{TiO}_{2}$ 纳米复合薄膜并进行 了表征. 前期已对薄膜光催化和正偏压下光电催化降解 有机染料的性能进行了报道 ${ }^{[16,17]}$, 本工作以罗丹明 $\mathrm{B}$ 为 模拟污染物, 对薄膜在负偏压和可见光作用下光催化降 解规律和机理进行探索. 旨在一方面了解 $\mathrm{TiO}_{2}$ 复合电 极在负偏压作用下的光催化降解特性, 另一方面为探索 可见光催化降解机理寻找更加有效的研究手段. 以复合 薄膜为电极, 在负偏压和可见光作用下研究光催化反应 的规律和机理, 在国内外鲜见报道.

\section{2 结果与讨论}

\section{1 外加负偏压对 $(\mathrm{Ni}-\mathrm{Mo}) / \mathrm{TiO}_{2}$ 薄膜电极光电催化降 解性能的影响}

图 1 是在可见光照射下, 对 $(\mathrm{Ni}-\mathrm{Mo}) / \mathrm{TiO}_{2}(\mathrm{Cu}$ 为基
片, 下同)薄膜施加不同负偏压光电催化降解罗丹明 $\mathrm{B}$ 的实验结果. 由图 1 可见, 外加偏压为 $-0.200 \mathrm{~V}$ 时, 薄 膜对染料的光电催化活性小于光催化活性(b 线的降解 率低于 $\mathrm{a}$ 线), 当外加负偏压的绝对值大于 $0.3 \mathrm{~V}$ 时, 薄 膜的光电催化活性大于光催化活性. 同时可见, 薄膜的 光电催化活性先随偏压绝对值的增大而增大, 而后又随 偏压绝对值的增大而减小, 偏压为 $-0.400 \mathrm{~V}$ 时光电催 化活性最大, 反应 $60 \mathrm{~min}$, 光电催化的最大降解率 (88.3\%)约为光催化降解率 $(44.3 \%)$ 的两倍.

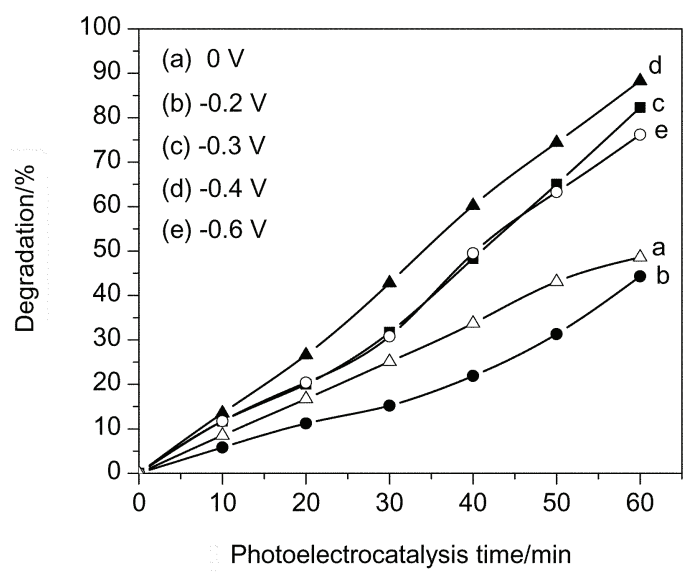

图 1 可见光照射下外加负偏压对 $(\mathrm{Ni}-\mathrm{Mo}) / \mathrm{TiO}_{2}$ 薄膜光电催化性能的 影响

Figure 1 Effect of cathodic bias on photoelectrocatalytic activity of $(\mathrm{Ni}-\mathrm{Mo}) / \mathrm{TiO}_{2}$ thin film under visible light irradiation

对光吸收國值在紫外光范围内的半导体光催化剂, 可见光照射下催化降解染料属于染料敏化光催化反 应 ${ }^{[12]}$. 首先染料分子吸收可见光被激发, 然后激发态的 染料分子向半导体的导带注入光电子, 从而引起染料分 子的解离. 以罗丹明 $\mathrm{B}(\mathrm{RhB})$ 为例, 上述过程可表示为: $\mathrm{RhB}+h v \rightarrow \mathrm{RhB}^{*} ; \mathrm{RhB}^{*} \rightarrow \mathrm{RhB}^{{ }^{+}+}+\mathrm{e}^{-}$. 注入到导带的光 电子迁移到催化剂的表面与溶液中的溶解氧作用生成 $\cdot \mathrm{O}_{2}^{-}$、・ $\mathrm{OH}$ 等可使罗丹明 $\mathrm{B}$ 氧化降解的活性物种. 基于 上述机理, 容易理解, 若关闭光源进行电催化降解, 应 该出现罗丹明 $\mathrm{B}$ 的降解率随电极负偏压绝对值的增大 而增大的规律. 本工作使用 $(\mathrm{Ni}-\mathrm{Mo}) / \mathrm{TiO}_{2}$ 薄膜电极 (与 P25 $\mathrm{TiO}_{2} / \mathrm{ITO}$ 光吸收阈值相同 ${ }^{[16]}$ )证实了这个结果(数据 从略), 显然, 这是电极表面活性氧化物种浓度随着电 极负偏压绝对值的增大而增大的缘故. 由此可以推测出, 图 1 中出现的不同于电催化的光电催化规律只能是光照 引起的. 考虑到罗丹明 $\mathrm{B}$ 是阳离子型染料, 负偏压有利 于染料在电极表面的吸附; 以及在较大负偏压下, 由激 发态染料分子解离生成的光生电子对活性氧化物种形 成的作用不大，这就预示着染料正离子自由基 $\mathrm{RhB}^{\circ}{ }^{+}$的 存在对光电催化降解反应乃至光催化反应有重要影响.

在负偏压条件下，含氧活性物种的生成反应与 $\mathrm{RhB}^{\cdot+}$ 的产生反应是竞争关系, 这可能是出现图 1 结果 的原因. 


\section{$2.2(\mathrm{Ni}-\mathrm{Mo}) / \mathrm{TiO}_{2}$ 薄膜与 $\mathrm{TiO}_{2} / \mathrm{ITO}$ 薄膜光电催化降解 性能的比较}

图 2 是 $(\mathrm{Ni}-\mathrm{Mo}) / \mathrm{TiO}_{2}$ 薄膜电极和用作比较的多孔 $\mathrm{P} 25 \mathrm{TiO}_{2} / \mathrm{ITO}$ 薄膜电极在可见光和偏压为 $-0.400 \mathrm{~V}$ 作 用下光电催化罗丹明 $\mathrm{B}$ 的降解率随反应时间的变化(两 薄膜的表面组成和状态、晶相结构和光谱特性见文献 [16]). 由图可见, 光电催化降解 $30 \mathrm{~min}$, 复合薄膜的降 解率(42.8\%) 是 $\mathrm{TiO}_{2} / \mathrm{ITO}$ 薄膜的 2.46 倍; 光电催化降解 $60 \mathrm{~min}$, 复合电极对罗丹明 B 降解率高达 $88.3 \%$, 它与 $\mathrm{TiO}_{2} / \mathrm{ITO}$ 电极的降解率之比仍能达到 1.56 倍. 由文献 [17]可知, 在可见光和最佳正偏压 $(0.2 \mathrm{~V}$, 与白光最佳偏 压相同)作用下 (其它光电催化条件与图 2 相同), $(\mathrm{Ni}-\mathrm{Mo}) / \mathrm{TiO}_{2}$ 薄膜光电催化降解罗丹明 B $60 \mathrm{~min}$, 降解 率只有 $70.1 \%$, 仅为相同条件下多孔 P25 $\mathrm{TiO}_{2} / \mathrm{ITO}$ 膜电 极的 1.15 倍. 由此可知, 在可见光和负偏压作用下, 复 合电极具有优良的光电催化性能, 并且远高于它在正偏 压下的光电催化活性.

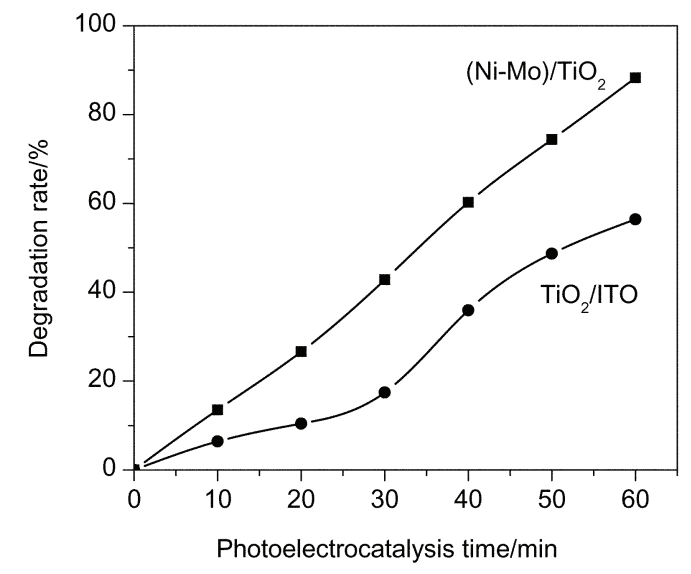

图 2 不同薄膜电极光电催化性能的比较

Figure 2 Dependence of Rhodamine B degradation rate on the nature of thin film electrode

\section{$2.3(\mathrm{Ni}-\mathrm{Mo}) / \mathrm{TiO}_{2}$ 薄膜光催化降解活性与外加负偏压 的依赖关系}

为了研究光的照射在降解中的作用, 本工作在一定 负偏压范围内对有可见光照射和无光照射条件下的降 解实验进行了对比研究. 图 3 是反应时间为 $50 \mathrm{~min}$ 时的 情况, 图中 $\Delta R$ 是光电催化和电催化的降解率之差, $\Delta I$ 是光电催化平均电流与电催化平均电流之差. 由图可知, $\Delta R$ 和 $\Delta I$ 随外加偏压的变化呈现了相似的变化规律, 它 们均先随外加负偏压绝对值的增加而增加, 而后又随偏 压绝对值的增加而减小, 在所测实验数据中偏压为 $-0.4 \mathrm{~V}$ 时两者同时出现最大值. 实际上, 由图 3 表达的 实验规律可以确定 $\Delta R$ 和 $\Delta I$ 最大值应在 $-0.3 \mathrm{~V}$ 和 -0.4 $\mathrm{V}$ 之间.

由前述 2.1 节对实验结果的分析可知, 导致相同负 偏压下光电催化和电催化的降解率不同的因素为: 在光

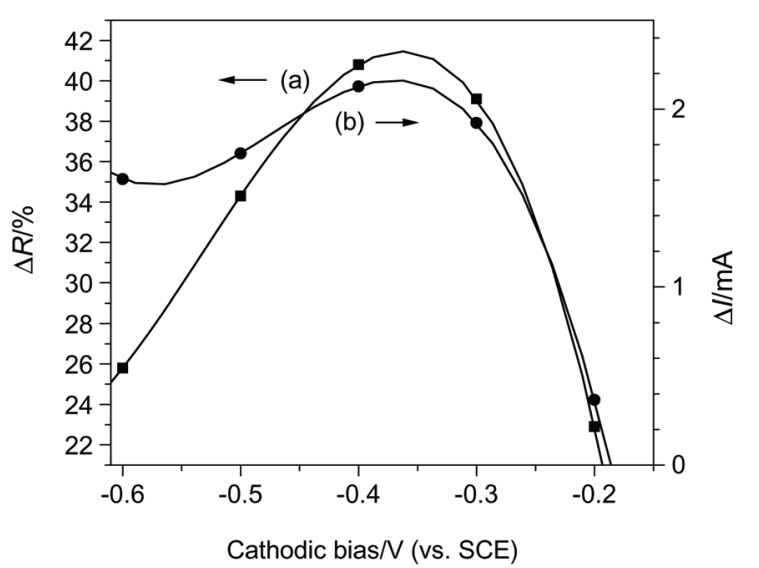

图 3 罗丹明 $\mathrm{B}$ 的电势扫描曲线

Figure 3 Cathodic bias scanning experiments of dye $\mathrm{RhB}$ from -0.2 $\mathrm{V}$ to $-0.6 \mathrm{~V}$. Plots (a) and (b) show the difference of the degradation rate and current intensity between the photoelectrochemical reaction and the corresponding electrolysis reaction, respectively

电催化反应体系中存在罗丹明 $\mathrm{B}$ 正离子自由基 $\left(\mathrm{RhB}^{\bullet+}\right)$, 而电催化反应体系中不存在. 即参与降解反应的被降解 物在两个反应体系的存在形式不相同. 当负偏压绝对值 较小时，尽管此时两反应体系中参与反应的罗丹明 $\mathrm{B}$ 有 不同形式，但是电极附近活性氧化物的浓度较小，因而 两者的降解率之差 $\Delta R$ 较小; 当负偏压绝对值较大时, 由于对 $\mathrm{RhB}^{*} \rightarrow \mathrm{RhB}^{\bullet+}+\mathrm{e}^{-}$的反应的阻碍作用很大，故 两反应体系中参与降解反应的罗丹明 $\mathrm{B}$ 均主要以 $\mathrm{RhB}$ 形式存在, 因而两者的 $\Delta R$ 也较小; 只有偏压适中时, 不 仅参与降解反应的染料以不同的形式存在，而且电极附 近活性氧化物种浓度较大，因而有明显的降解率之差. 这就解释了图 3(a)线表示的规律.

$\Delta I$ 随外加负偏压的变化规律也容易理解. 实验中 测定的光电催化电流和电催化电流都是由外电路迁移 到电极表面的电子与溶解氧之间的反应引起的，此反应 可表示为:

$$
\mathrm{O}_{2}+\mathrm{e}^{-} \rightarrow \cdot \mathrm{O}_{2}^{-} / \cdot \mathrm{OH}
$$

由于光照下的光电催化体系中参与反应的罗丹明 $\mathrm{B}$ 以 $\mathrm{RhB}$ 和 $\mathrm{RhB}^{\bullet+}$ 两种形式存在, 而电催化体系中只有 $\mathrm{RhB}$, 由此可能导致后续反应的不同, 即光电催化主反 应为:

$\cdot \mathrm{O}_{2}^{-} / \cdot \mathrm{OH}+\mathrm{RhB}^{\bullet+} \rightarrow$ degradation products

电催化反应为:

$\cdot \mathrm{O}_{2}^{-} / \cdot \mathrm{OH}+\mathrm{RhB} \rightarrow$ degradation products

若(2)反应的速率大于 (3)反应，则必然致使光电化 学体系发生的(1)反应的速率大于电催化体系, 由此导 致 $\Delta I>0$. 在负偏压绝对值较小时, 两反应体系的活性 氧化物种浓度都较低, 而当负偏压绝对值较大时, 两反 
应体系的 $\mathrm{RhB}^{-}+$的浓度很低或不存在，这都会导致两反 应体系的后继反应差别不大, 从而使 $\Delta I$ 较小. 只有在适 中的负偏压下, 由于 $\mathrm{RhB}^{-}{ }^{+}$和活性氧化物物种浓度都较 大, 才会使 $\Delta I$ 较大, 这就是图 3 曲线(b)出现极大值的原 因.

总之, 图 3 的实验结果可以证明, 罗丹明 $\mathrm{B}$ 通过正 离子自由基 $\mathrm{RhB}^{-}+$与活性氧化物作用而被降解是 $(\mathrm{Ni}-\mathrm{Mo}) / \mathrm{TiO}_{2}$ 薄膜光电催化(或光催化)的主要反应途径.

\section{4 反应体系中主要活性氧化物种的确定}

对可见光和负偏压作用下的光电催化或光催化染 料降解反应, 主要可能的活性氧化物种为 $\cdot \mathrm{O}_{2}^{-}$和 $\cdot \mathrm{OH}^{[20]}$, 为进一步探索它们的作用机制, 本工作进行了相关探索.

图 4 是在可见光照射和外加偏压为 $-0.4 \mathrm{~V}$ 时, 在 催化体系中加入不同活性物种捕获剂的实验结果. 以 $\mathrm{AgNO}_{3}$ 为电子捕获剂、以苯醌作为超氧自由基 $\left(\cdot \mathrm{O}_{2}^{-}\right)$捕 获剂、叔丁醇作为羟基自由基 $(\cdot \mathrm{OH})$ 捕获剂, 实验中各种 捕获剂在反应体系中的初始浓度均与模拟污染物罗丹 明 $\mathrm{B}$ 的初始浓度相同, 即体积摩尔浓度为 $1.04 \times 10^{-5}$ $\mathrm{mol} \cdot \mathrm{L}^{-1}$, 其它光电催化条件与 4.2 节相同.

作者认为, 以反应时间为 $40 \sim 50 \mathrm{~min}$ 的数据进行分 析较为合理, 这是因为光电催化时间过短, 不同实验降 解率的差别不能充分表现出来; 时间过长, 一方面加入 的各种捕获剂消耗较多, 另一方面被降解物有浓度效应, 这都会掩盖实验的实质. 本文以反应时间 $50 \mathrm{~min}$ 的数据 进行分析. 由图 4 可见, 加入羟基自由基捕获剂使光电 催化罗丹明 $\mathrm{B}$ 的降解率明显降低(图中 $\mathrm{d}$ 线), 与不加任 何捕获剂相比较(图中 $\mathrm{b}$ 线), 光催化 $50 \mathrm{~min}$, 降解率由 $74.4 \%$ 降低为 $54.5 \%$, 降低值为 $19.9 \%$. 图 4 中 $\mathrm{c}$ 线是向 反应体系中加入超氧自由基 $\left(\cdot \mathrm{O}_{2}^{-}\right)$捕获剂的实验结果, 光电催化相同时间, 染料的降解率为 $65.5 \%$, 相对于不 加任何捕获剂体系, 降解率只降低了 $8.9 \%$. 由此可知, 在该光电催化条件下, $\cdot \mathrm{OH}$ 是降解罗丹明 $\mathrm{B}$ 的主要活性 物种. 图中曲线(a)是向反应体系中加入电子捕获剂的 实验结果. 由图可知, 催化降解 $50 \mathrm{~min}$, 染料降解率为 $91.5 \%$ ，与不加捕获剂的体系相比，降解率不仅没有降 低, 反而提高了 $17.1 \%$. 这个结果可以由上述图 3 进行 解释. 由图 3 可见, 复合电极光催化活性最大值出现在 偏压为 $-0.3 \sim-0.4 \mathrm{~V}$ 之间. 容易理解, 在恒定电极偏 压为 $-0.4 \mathrm{~V}$ 时加入电子捕获剂(图 4), 与不加电子捕获 剂条件下由偏压为 $-0.4 \mathrm{~V}$ 继续小幅度正移偏压(图 3)的 作用是相同的, 因而会出现图 4 中加入电子捕获剂后罗 丹明 $\mathrm{B}$ 降解率增加的结果. 显然, 这个实验进一步证实 了可见光照射下光电催化降解染料的反应中, 染料正离 子自由基的存在有重要作用.

此外, 我们还对可见光照射下光催化罗丹明 B 的体 系进行了活性物种的测定, 测定方法与上述光电催化相 同, 光催化实验条件除不加偏压外其它也与 4.2 节所述 的光电催化条件相同.

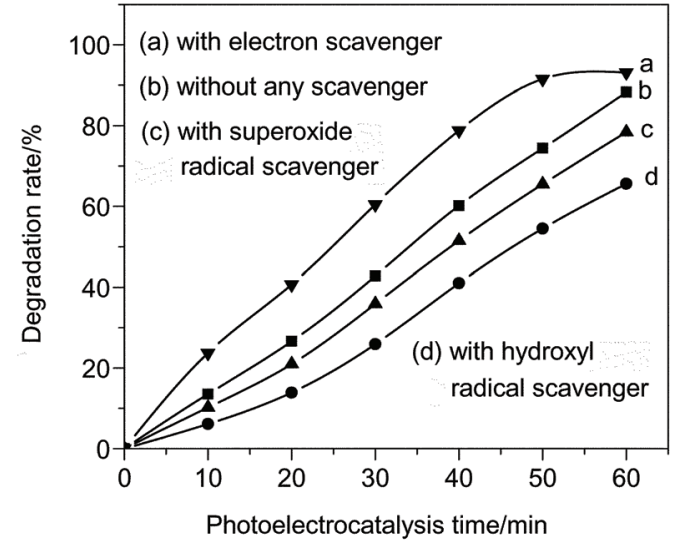

图 4 可见光照射下不同捕获剂对 $(\mathrm{Ni}-\mathrm{Mo}) / \mathrm{TiO}_{2}$ 薄膜光电催化活性的 影响

Figure 4 Effect of different scavengers on the photoelectrocatalytic activity of $(\mathrm{Ni}-\mathrm{Mo}) / \mathrm{TiO}_{2}$ thin film under visible light irradiation (The applied cathodic bias is $-0.4 \mathrm{~V}$ )

测定结果如图 5 所示. 容易看出, $\cdot \mathrm{O}_{2}^{-}$是光催化降 解罗丹明 B 的主要活性物种, 这与我们前面所作研究工 作 ${ }^{[16]}$ (降解刚果红)的结论相一致. 作者认为, 光电催化 和光催化体系主要活性物种的不同是膜电极上的偏压 引起的, 对光电催化体系, 较高的负偏压意味着电极上 有较负的电势, 它有利于将 $\cdot \mathrm{O}_{2}^{-}$按下式转化为 $\cdot \mathrm{OH}$ :

$$
\cdot \mathrm{O}_{2}^{-}+\mathrm{e}^{-}+2 \mathrm{H}^{+} \rightarrow \mathrm{H}_{2} \mathrm{O}_{2} ; \mathrm{H}_{2} \mathrm{O}_{2}+\mathrm{e}^{-} \rightarrow \cdot \mathrm{OH}+\mathrm{OH}^{-}
$$

这个结果表明，可以通过电极电势调控电极表面附 近活性物种的种类和浓度以及被降解物的存在形式, 从 而对光催化机制进行探讨.

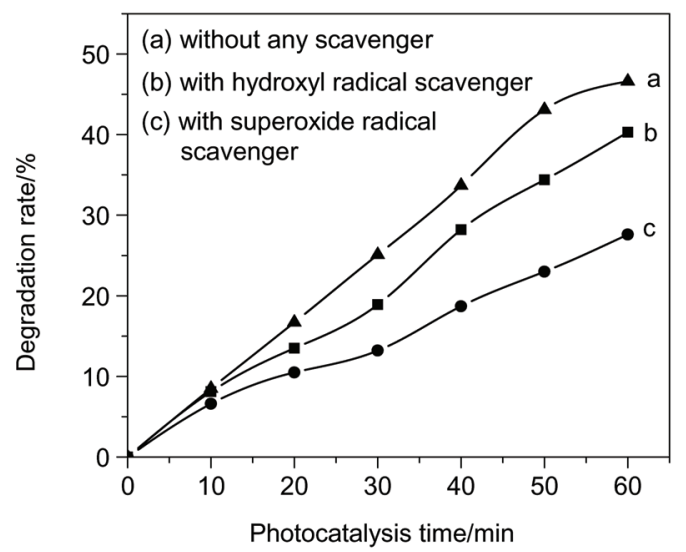

图 5 可见光照射下不同捕获剂对 $(\mathrm{Ni}-\mathrm{Mo}) / \mathrm{TiO}_{2}$ 薄膜光催化活性的影 响

Figure 5 Effect of different scavengers on the photocatalytic activity of $(\mathrm{Ni}-\mathrm{Mo}) / \mathrm{TiO}_{2}$ thin film under visible light irradiation

\section{5 薄膜对溶解氧的光电化学活性}

为了揭示 $(\mathrm{Ni}-\mathrm{Mo}) / \mathrm{TiO}_{2}$ 薄膜电极在负偏压下的光电 催化机理和光电催化活性提高的原因, 对薄膜催化还原 溶解氧的能力进行了线性电势扫描伏安(LSV)测定. 为 
了便于比较, 对 $\mathrm{TiO}_{2} / \mathrm{ITO}$ 薄膜也进行了测量. 实验在室 温 $\left(25^{\circ} \mathrm{C}\right)$ 下进行, 使用 $\mathrm{H}$ 型硬质玻璃电解槽, 以 $(\mathrm{Ni}-\mathrm{Mo}) / \mathrm{TiO}_{2}$ 薄膜或 $\mathrm{TiO}_{2} / \mathrm{ITO}$ 薄膜为工作电极, $\mathrm{Pt}$ 片电 极为辅助电极, 饱和甘录电极为参比电极. 工作电极的 受光面积为 $1 \mathrm{~cm}^{2}$, 测试溶液为 $\mathrm{pH}$ 值等于 2 、浓度为 0.5 $\mathrm{mol} / \mathrm{L}$ 的 $\mathrm{Na}_{2} \mathrm{SO}_{4}$ 溶液, 实验前通入纯 $\mathrm{O}_{2}$, 使测试溶液为 大气压力下的饱和氧溶液, 用辰华 $\mathrm{CHI} 660 \mathrm{C}$ 电化学工 作站进行测定, 扫描速率为 $50 \mathrm{mV} / \mathrm{s}$, 由高电势至低电 势进行扫描. 以 $150 \mathrm{~W}$ 卤钨灯为光源, 不滤光, 其它条 件同 4.2 节.

实验结果如图 6 所示, 由图可知, $\mathrm{TiO}_{2} / \mathrm{ITO}$ 薄膜电 极的电流响应远小于 $(\mathrm{Ni}-\mathrm{Mo}) / \mathrm{TiO}_{2}$ 电极. 图中曲线(a)是 $\mathrm{TiO}_{2} / \mathrm{ITO}$ 薄膜在暗态下的伏安曲线, 电极电势为 $-0.743 \mathrm{~V}$ 的峰位对应于溶解氧在 $\mathrm{TiO}_{2}$ 表面上的还原反 应 $^{[21]}$, 峰电流密度为 $1.13 \mathrm{~mA} / \mathrm{cm}^{2}$. 曲线(b)是 $\mathrm{TiO}_{2} / \mathrm{ITO}$ 电极在光照下的伏安曲线, 峰电势为 $-0.717 \mathrm{~V}$, 相对于 暗态正移了 $26 \mathrm{mV}$; 峰电流密度为 $1.14 \mathrm{~mA} / \mathrm{cm}^{2}$, 相对 于暗态只增加了 $0.01 \mathrm{~mA} / \mathrm{cm}^{2}$, 光响应不大. 图 6 中曲线 (c)和(d) 分别是 $(\mathrm{Ni}-\mathrm{Mo}) / \mathrm{TiO}_{2}$ 电极在暗态和光照下的线 性电势扫描伏安曲线, 暗态和光照下溶解氧还原的峰电 势分别为 $-0.100 \mathrm{~V}$ 和 $-0.096 \mathrm{~V}$, 两者相差不大; 峰电 流密度分别是 $2.91 \mathrm{~mA} / \mathrm{cm}^{2}$ 和 $4.83 \mathrm{~mA} / \mathrm{cm}^{2}$, 光响应明显.

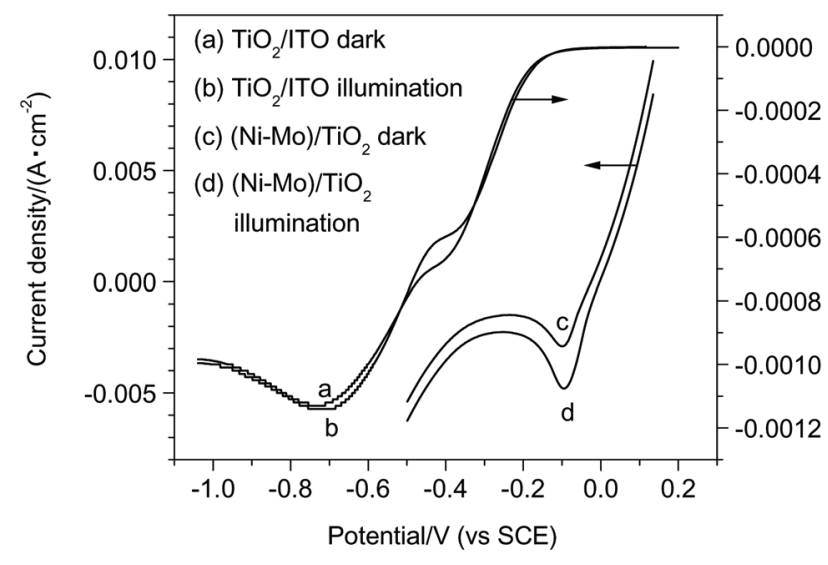

图 $6(\mathrm{Ni}-\mathrm{Mo}) / \mathrm{TiO}_{2}$ 和 $\mathrm{TiO}_{2} / \mathrm{ITO}$ 电极线性电势扫描伏安曲线

Figure 6 Linear potential sweep voltammograms of the $(\mathrm{Ni}-\mathrm{Mo}) / \mathrm{TiO}_{2}$ and $\mathrm{TiO}_{2} / \mathrm{ITO}$ electrodes

由上述测定结果可知, 光作用下 $(\mathrm{Ni}-\mathrm{Mo}) / \mathrm{TiO}_{2}$ 薄膜 电极相对于 $\mathrm{TiO}_{2} / \mathrm{ITO}$ 电极对溶解氧还原的电极电势正 移了 $0.621 \mathrm{~V}$; 复合薄膜的光电流密度是 $\mathrm{TiO}_{2} / \mathrm{ITO}$ 薄膜 的 4.2 倍. 由此可以得出: $(\mathrm{Ni}-\mathrm{Mo}) / \mathrm{TiO}_{2}$ 薄膜是氧气光催 化还原优良的催化剂.

\section{$2.6(\mathrm{Ni}-\mathrm{Mo}) / \mathrm{TiO}_{2}$ 薄膜的光电催化降解机理}

$(\mathrm{Ni}-\mathrm{Mo}) / \mathrm{TiO}_{2}$ 电极的扫描电子显微镜、 $\mathrm{X}$ 射线衍射、 拉曼光谱和紫外-可见漫反射光谱测试结果表明 ${ }^{[16]}$ : $(\mathrm{Ni}-\mathrm{Mo}) / \mathrm{TiO}_{2}$ 薄膜由 $\mathrm{TiO}_{2}$ 纳米粒子相和纳米晶 $\mathrm{Ni}-\mathrm{Mo}$ 固溶体相构成, 薄膜中 $\mathrm{TiO}_{2}$ 纳米微粒的晶相结构、原子
的空间排布及其相互作用并未受到基质 Ni-Mo 合金明 显的作用. Ni-Mo 的存在不仅未使薄膜中 $\mathrm{TiO}_{2}$ 的带隙发 生改变, 而且吸光强度也未增加; $\mathrm{TiO}_{2}$ 微粒均匀镶嵌在 $\mathrm{Ni}-\mathrm{Mo}$ 合金中，并且以 $50 \sim 100 \mathrm{~nm}$ 的粒径裸露于薄膜 表面. 这种结构一方面使得 $\mathrm{TiO}_{2}$ 微粒与合金以较大的 界面充分接触, 有效形成 Schottky 异质结, 有助于 $\mathrm{TiO}_{2}$ 中的光生电子落入金属势阱中; 另一方面 Ni-Mo 合金对 光电子起到了集电极的作用, 为光电子收集和输运提供 了储存器和良好电子通道. 2.5 节实验结果表明, 纳米晶 Ni-Mo 对溶解氧有优异的催化还原作用, 这在反应动力 学上有利于含氧活性物种的产生以及 $\mathrm{RhB}^{*} \rightarrow \mathrm{RhB}^{\bullet+}+$ $\mathrm{e}^{-}$反应速率的提高. 总之, 上述三种作用是 $(\mathrm{Ni}-\mathrm{Mo}) /$ $\mathrm{TiO}_{2}$ 薄膜光电催化降解活性提高的重要原因.

由于可见光不能使复合电极中的 $\mathrm{TiO}_{2}$ 产生光生载 流子, 因而在所加偏压不足以发生电激发的一般可见光 电催化反应中, 只存在使污染物降解的阴极(导带)活性 物种, 这与紫外光作用下的光电催化反应存在阴极和阳 极(导带和价带)两类活性物种有本质的不同. 在可见光 作用下施加阴极偏压, 将使 $\mathrm{O}_{2}$ 与电子间的还原反应[反 应(1)或图 7 中步骤(9)、(11)和(12)]的活化吉布斯自由能 减小 (反应速率常数增大), 而激发态的染料解离生成染 料正离子自由基和电子的氧化反应[可视为下述反应(6) 和(7)的加和, 见图 7 中步骤(6)和(7)]的活化吉布斯自由 能增大 ${ }^{[22]}$ (反应速率常数减小). 这有利于阴极活性氧化 物物种、特别是・ $\mathrm{OH}$ 的生成，而对染料正离子自由基的 产生有抑制作用. 生成活性氧化物种所需要的电子一部 分来源于外电路 [见图 7 中的步骤(8)], 另一部分来源于 激发态染料解离产生的光电子 [见图 7 中步骤(6)和(7)]. 在可见光作用下施加阳极偏压, 则使上述生成活性氧化 物种的还原反应[反应(1)]的活化吉布斯自由能增大，激 发态染料解离的氧化反应的活化吉布斯自由能减小. 这 势必加速染料正离子自由基和光电子的生成而降低反 应(1)的生成速率. 此时生成活性氧化物种所需要的电 子只是光电子的一部分, 而剩余的另一部分光电子则流 出外电路. 此外, 由上述 2.4 节可知, 负偏压下光催化罗 丹明 $\mathrm{B}$ 的主要活性物种为 $\cdot \mathrm{OH}$, 光催化降解该染料的主 要活性物种为 $\cdot \mathrm{O}_{2}^{-}$, 由此结果及该节中相关的分析不难 推测出, 正偏压下主要的活性物种应为 $\cdot \mathrm{O}_{2}^{-}$.

由本文以及文献[12]和[16]可知, 可见光催化降解 染料的主要反应途径为染料正离子自由基与活性氧化 物种之间的反应. 由上述讨论可知，偏压对两者浓度的 影响是相反的，不管是正偏压还是负偏压，两者的浓度 不可能同时达到最大值, 这是可见光催化反应中无论施 加阳极偏压还是阴极偏压, 都存在一个最佳中间值的主 要原因. 对可见光催化降解罗丹明 $\mathrm{B}$ 反应而言, 负偏压 与正偏压作用下主要反应途径的不同在于, 前者的主要 活性物种为 $\cdot \mathrm{OH}$, 而后者为 $\cdot \mathrm{O}_{2}^{-}$, 由于 $\cdot \mathrm{OH}$ 的氧化能力 远大于 $\bullet \mathrm{O}_{2}^{-}\left(\varphi^{\ominus}\left(\cdot \mathrm{OH} / \mathrm{H}_{2} \mathrm{O}, \mathrm{H}^{+}\right)=2.72 \mathrm{~V} ; \varphi^{\ominus} \quad\left(\mathrm{HO}_{2} / \mathrm{H}_{2} \mathrm{O}_{2}\right)\right.$ 


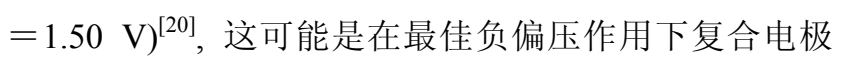
的光催化活性大于最佳正偏压下活性的主要原因. 此外, 在负偏压作用下有利于阳离子型染料罗丹明 B 在催化 剂表面的吸附, 也可能是催化活性较高的原因之一.

综合本文的探索和当前人们的认识 ${ }^{[23,24]}$, 提出在负 偏压和可见光作用下光电催化降解罗丹明 $\mathrm{B}$ 的反应机 理为:

$$
\begin{aligned}
& \mathrm{RhB}+h v \rightarrow \mathrm{RhB}^{*} \\
& \mathrm{RhB}^{*}+(\mathrm{Ni}-\mathrm{Mo}) / \mathrm{TiO}_{2} \rightarrow(\mathrm{Ni}-\mathrm{Mo}) / \mathrm{TiO}_{2}\left(\mathrm{e}^{-}\right)+\mathrm{RhB}^{\bullet+} \\
& (\mathrm{Ni}-\mathrm{Mo}) / \mathrm{TiO}_{2}\left(\mathrm{e}^{-}\right) \rightarrow(\mathrm{Ni}-\mathrm{Mo})\left(\mathrm{e}^{-}\right) / \mathrm{TiO}_{2} \\
& \text { External circuit }\left(\mathrm{e}^{-}\right) \rightarrow(\mathrm{Ni}-\mathrm{Mo})\left(\mathrm{e}^{-}\right) / \mathrm{TiO}_{2} \\
& \mathrm{O}_{2}+(\mathrm{Ni}-\mathrm{Mo})\left(\mathrm{e}^{-}\right) / \mathrm{TiO}_{2} \rightarrow \cdot \mathrm{O}_{2}^{-}+(\mathrm{Ni}-\mathrm{Mo}) / \mathrm{TiO}_{2} \\
& \bullet \mathrm{O}_{2}^{-}+\mathrm{RhB}^{\bullet+} \rightarrow \operatorname{degradation~}^{-} \text {roducts (slow) } \\
& \bullet \mathrm{O}_{2}^{-}+\mathrm{e}^{-}+2 \mathrm{H}^{+} \rightarrow \mathrm{H}_{2} \mathrm{O}_{2} \text { (fast) } \\
& \mathrm{H}_{2} \mathrm{O}_{2}+\mathrm{e}^{-} \rightarrow \cdot \mathrm{OH}+\mathrm{OH}^{-} \text {(fast) } \\
& \bullet \mathrm{OH}+\mathrm{RhB}^{\bullet+} \rightarrow \text { degradation products (fast) }
\end{aligned}
$$

式中 $\mathrm{RhB} *$ 表示罗丹明 $\mathrm{B}$ 的电子激发态, $\mathrm{RhB}^{\bullet+}$ 表示罗丹 明 B 自由基. (5)式表示罗丹明 B 吸收可见光被激发, (6) 式表示电子从罗丹明 $\mathrm{B}$ 的激发态跳入复合薄膜中 $\mathrm{TiO}_{2}$ 的导带, (7)式则表示复合薄膜中 $\mathrm{TiO}_{2}$ 的导带电子进入 Ni-Mo 中. 为便于理解, 将上述机理示意于图 7 中.

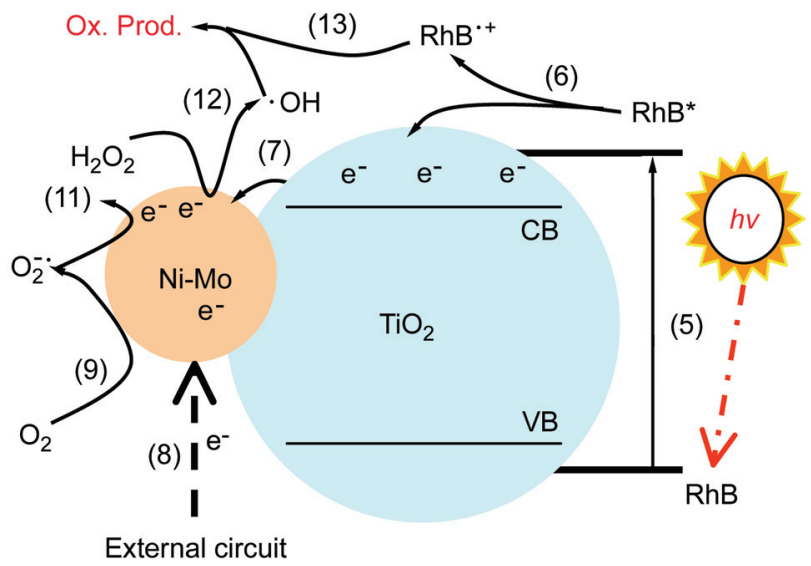

图 7 可见光和负偏压作用下 $(\mathrm{Ni}-\mathrm{Mo}) / \mathrm{TiO}_{2}$ 膜电极光电催化降解罗丹 明 B 的机理示意图

Figure 7 Schematic diagram of the mechanism for the photoelectrocatalytic action of $(\mathrm{Ni}-\mathrm{Mo}) / \mathrm{TiO}_{2}$ thin film on Rhodamine $\mathrm{B}$ at negative bias under visible light

\section{3 结论}

在负偏压和可见光作用下, $(\mathrm{Ni}-\mathrm{Mo}) / \mathrm{TiO}_{2}$ 薄膜具有 优异的光催化降解罗丹明 $\mathrm{B}$ 的性能. 复合薄膜对溶解氧
和激发电子优良的催化还原作用是光电催化降解活性 提高的重要原因. 在复合薄膜电极与溶液界面间同时生 成的羟基自由基和染料正离子自由基对染料的光电催 化降解有决定性作用. 光电化学与电化学相结合的技术 是研究可见光催化降解染料反应机制的一种有效方法.

\section{4 实验部分}

\section{1 薄膜电极的制备和表征}

实验所用试剂均为分析纯, $\mathrm{TiO}_{2}$ 是 P-25 纳米粉(德 国 Degussa 公司生产, $80 \%$ 锐钛矿相, $20 \%$ 金红石相). $(\mathrm{Ni}-\mathrm{Mo}) / \mathrm{TiO}_{2}$ 薄膜电极和用作比较的多孔 P25 $\mathrm{TiO}_{2} / \mathrm{ITO}$ 薄膜电极的制备方法与文献[16]相同, 本文不再陈述.

用日本理学 $D / m a x-R B$ 型 $X$ 射线衍射仪(X-ray diffractometer, XRD)分析薄膜的结构, $\mathrm{Cu} \mathrm{K \alpha}$ 辐射, 掠角 为 $3^{\circ}$. 使用 LEO1530 型热场发射扫描电镜(scanning electron microscope, SEM) 观察薄膜的表面形貌. 用 Oxford 7426 型能谱仪(energy dispersive spectrometer, EDS)测定薄膜表面的组成. 用 JASCO V-570 型紫外-可 见-近红外分光光度计测定紫外-可见(ultraviolet-visible, UV-Vis)漫反射光谱. 使用英国 Renishaw 公司生产的 inVia 型拉曼光谱仪测定薄膜的拉曼光谱，激发波长 514 $\mathrm{nm}$.

\section{2 光电催化实验}

室温 $\left(25{ }^{\circ} \mathrm{C}\right)$ 下，将 $14 \mathrm{~mL}$ 浓度为 $5.0 \mathrm{mg} / \mathrm{L} 、 \mathrm{pH}=2$ 的罗丹明 $\mathrm{B}$ 溶液(其中含支持电解质 $\mathrm{Na}_{2} \mathrm{SO}_{4}$ 浓度为 0.5 $\mathrm{mol} / \mathrm{L}$ ) 注入硬质玻璃反应器内, 以复合薄膜 (或 $\mathrm{TiO}_{2} / \mathrm{ITO}$ 薄膜) 为工作电极, $\mathrm{Pt}$ 片为辅助电极, 饱和甘录 电极(saturated calomel electrode, $\mathrm{SCE}$ )为参比电极构成 三电极系统. $(\mathrm{Ni}-\mathrm{Mo}) / \mathrm{TiO}_{2}$ 薄膜(或 $\mathrm{TiO}_{2} / \mathrm{ITO}$ 薄膜)与溶 液的接触面积为 $1 \mathrm{~cm} \times 2 \mathrm{~cm}$. 外加偏压通过 HDV-7 型 恒电位仪施加. 用 $150 \mathrm{~W}$ 卤铇灯为光源, 通过滤光片得 到最小波长为 $420 \mathrm{~nm}$ 的可见光，薄膜与光源距离为 $18.5 \mathrm{~cm}$, 氧气流量为 $50 \mathrm{~mL} / \mathrm{min}$. 样品放入溶液中 20 min 后开始实验，每间隔 $10 \mathrm{~min}$ 取样一次，用 721 型分 光光度计(波长 $552 \mathrm{~nm}$ ) 测定罗丹明 $\mathrm{B}$ 溶液的吸光度，并 以此计算罗丹明 $\mathrm{B}$ 的降解率.

\section{References}

[1] Ishibashi, K.; Fujishima, A.; Watanabe, T.; Hashimoto, K. J. J. Photochem. Photobiol. A-Chem. 2000, 134, 139.

[2] Ohno, T.; Tokieda, K.; Higashida, S.; Matsumura, M. Appl. Catal. A-Gen. 2003, 244, 383

[3] Colon, G.; Maicu, M.; Hidalgo, M. C.; Navío, J. A.; Kubacka, A. J. Mol. Catal. A-Chem. 2010, 320, 14.

[4] Esquivel, K.; Arriaga, L. G.; Rodriguez, F. J.; Martinez, L.; Godinez, L. A. Water Res. 2009, 43, 3593.

[5] Hosseini, Z.; Taghavinia, N.; Sharifi, N.; Chavoshi, M.; Rahman, M. J. Phys. Chem. C 2008, 121, 18686.

[6] Ramírez-Meneses, E.; García-Murillo, A.; Carrillo-Romo, F. d. J.; García-Alamilla, R. Sol-Gel Sci. Technol. 2009, 52, 267.

[7] Liu, S. Y.; Wu, L. D.; Zhao, Z. X.; Feng, Q. G.; Wang, X.; Yang, C. D. J. Inorg. Mater. 2009, 24, 902. (刘少友, 吴林东, 赵钟兴, 冯庆 
革，王翔，杨朝德，无机材料学报，2009, 24, 902.)

[8] Zanoni, M.; Sene, J.; Anderson, M. A. J. Photochem. Photobiol. A: Chem. 2003, 157, 55 .

[9] Li, J. Q.; Li, L. P.; Zheng, L.; Xian, Y. Z.; Jin, L. T. Electrochim. Acta 2006, 51, 4949 .

[10] Vinodgopal, K.; Hotchandani, S.; Kamat, P. V. J. Phys. Chem. 1993, 97, 9040

[11] Rothenberger, G.; Fitzmaurice, D.; Gratzel, M. J. Phys. Chem. 1992 , 96, 5983

[12] Yang, J.; Dai, J.; Zhao, J. C.; Miu, J. Chin. Sci. Bull. 2009, 54, 2196. (杨娟, 戴俊, 赵进才, 缪娟, 科学通报, 2009, 54, 2196.)

[13] Yang, J.; Chen, C.; Ji, H.; Ma, W. H.; Zhao, J. C. J. Phys. Chem. B 2005, 109, 21900.

[14] Yang, J.; Miu, J.; Dai, J.; Li, J. T. J. Henan Polytechnic Univ. 2010, 29, 259. (杨娟, 缪娟, 戴俊, 李建通, 河南理工大学学报, 2010 , 29,259 .)

[15] Ji, H. W.; Ma, W. H.; Huang, Y. P.; Zhao, J. C.; Wang, Z. P. Chin. Sci. Bull. 2003, 48, 2199. (籍宏伟, 马万红, 黄应平, 赵进才, 王 正平, 科学通报, 2003, 48,2199 .)

[16] Li, A. C.; Li, G. H.; Zheng, Y.; Feng, L. L.; Zheng, Y. J. Acta Phys.-Chim. Sin. 2012, 28, 457. (李爱昌, 李桂花, 郑琰, 冯玲玲, 郑彦俊, 物理化学学报, 2012, 28, 457.)
[17] Li, A. C.; Lu, Y. H.; Cao, G. L.; Liu, P.; Liu, Z. R. J. Chin. Ceram. Soc. 2011, 39, 1361. (李爱昌, 卢艳红, 曹国蕾, 刘萍, 刘增仁, 硅 酸盐学报, 2011, 39, 1361.)

[18] Fan, C. L.; Priron, D. L.; Sieb, A.; Paradis, P. J. Electrochem. Soc. 1994, 141,382

[19] Mei, Y.; Jia, Z. B.; Cao, J. L.; Han, M. J.; Zhang, Y. F.; Wei, Y. Acta Energiae Solaris Sinica 2002, 23, 199. (梅燕，贾枕斌，曹江林，韩 梅娟, 张艳峰, 魏雨, 太阳能学报, 2002, 23, 199.)

[20] Xu, Y. M. Prog. Chem. 2009, 21, 524. (许宜铭, 化学进展, 2009, 21,524 .)

[21] Peralta-Hernández, J. M.; Manríquez, J.; Meas-Vong, Y. J. Hazard. Mater. 2007, 147, 588.

[22] Guo, H. T.; Qin, Q. X. Electrochemistry, Tianjin University Press, Tianjin, 2000, pp. 182 184. (郭鹤桐, 覃奇贤, 电化学教程, 天津 大学出版社, 天津, 2000, pp. 182 184.)

[23] You, X. F.; Chen, F.; Zhang, J. L.; Huang, J. Z.; Zhang, L. Z. Chin. J. Catal. 2006, 27, 270. (尤先锋, 陈锋, 张金龙, 黄家桢, 张利中, 催化学报, 2006, 27, 270.)

[24] Han, S. T.; Xi, H. L.; Shi, R. X.; Fu, X. Z.; Wang, X. X. Chin. J. Chem. Phys. 2003, 16, 339. (韩世同，习海玲，史瑞雪，付贤智，王 绪绪, 化学物理学报, 2003, 16, 339.) 